\title{
ARTICLES
}

\section{THE FUTURE OF TELEMEDICINE IN MODERN HEALTHCARE}

Mrs. Merlin Samuel John*

*Manager, Operations Network and Business Development Manager at Whealth International, Dubai. DOI: http://doi.org/10.47211/trr.2021.v07i01.003

\begin{abstract}
The COVID-19 has brought a tremulous shift in healthcare delivery. Since February 2020, telemedicine grew from 1\% of the primary care visit to nearly 43.5\% in April 2020. The report published in 2020 by Telemedicon, revealed that non-metro cities recorded a growth of $7 X$ in online consultations as compared to last year and started adopting an online mode of consultations amid the pandemic in metro to non-metro is 60:40 in 2020, as compared to 75:25 in 2019. This rapid growth presents telemedicine as the next frontier in healthcare. Digital and communication technologies have revolutionized the delivery of healthcare services. Telehealth is set to change the future of healthcare and remove many of the obstacles and challenges the patients and the industry face. Patients are rapidly transitioning to telehealth, $76 \%$ responded to the survey that they want to use telehealth going forward. Benefits of Telemedicine include convenience, access to care, better patient outcomes, a more efficient healthcare system, fewer hospital admissions, and fewer hospital re-admissions. With telemedicine's current trajectory and rapid adoption rate, it has the potential to disrupt and redefine the way health systems operate, deliver care, manage costs, setting the stage for a vastly different healthcare experience in the future. The widespread use of telemedicine helps to push the industry to make better platforms that can cooperate and communicate. Telemedicine is an effective way to alleviate the congestion of hospitals, improve the utilization of medical resources, and also a powerful tool that makes healthcare accessible, cost-effective, and significantly enhances patient engagement.
\end{abstract}

Key Words: Telemedicine, Telemedicine, healthcare, patient engagement.

\section{ABOUT AUTHOR:}

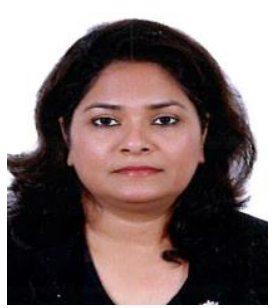

Author Mrs. Merlin Samuel John is the Operations, Network and Business Development Manager at Whealth International, Dubai, with more than 20 years of experience in the insurance and healthcare industry. 


\section{INTRODUCTION}

The Future of Telemedicine has limitless possibilities!

We have been talking about telehealth for nearly 30 years. But in the year 2020, the need for virtual health services has escalated globally. Before the COVID-19 pandemic, telehealth was more of a stretch goal than a reality for most providers despite the seeming prevalence of video conferencing. India's healthcare ecosystem is on the cusp of a paradigm flux. After remaining resistant to reform for the longest time, the sector is now open to innovation and digital technology-driven medical assistance that became a necessity amid pandemic. According to the recently published report titled "Reinventing Healthcare Delivery with Telemedicine" by Telemedicine Society of India (TSI) and Practo, India has recorded a $3 \mathrm{X}$ increase in the number of people using online consultations, while in-person appointments have reduced by 32 percent in between March to November 2020. The more the viral strain confined people to their homes, the more virtual care became a reality. According to the survey made in April 2020 by McKinsey, the consumer adoption of telehealth has skyrocketed with almost 70\% of in-person visits cancelled in the United States. Due to Covid-19, there has been a curious uptick among people to consult the doctor over a video call, seek diagnosis virtually, and order medicines online which in turn helped in decreasing the transmission rate of Covid-19 and limiting exposure to patients. Telemedicine has offered 5 C's: Care, Convenience, Comfort, Confidentiality, and Contagion.

\section{DEFINITION}

According to American Telemedicine Association, "Telemedicine is the remote delivery of health care services and clinical information using telecommunication technology. This includes a wide array of clinical services using internet, wireless, satellite and telephone media".

According to WHO, "Delivery of healthcare services, where distance is a critical factor, by all healthcare professionals using information and communication technology for the exchange of valid information for the diagnosis, treatment, and prevention of diseases and injuries, research and evaluation, and for continuing education of healthcare providers, all in the interests of advancing the health of individuals and their communities."

\section{TYPES}

There are three main types of telemedicine

* Real-Time Telemedicine: Also called Live Telemedicine. It includes any two-way communications using video conferencing and telephone consultations that allow the patient and providers to communicate in real-time. Assessments of medical history, basic visual examinations, psychiatric evaluations, and even Ophthalmic tests can be done in real-time.

* Remote Patient Monitoring (RPM): This allows the healthcare providers to monitor patient's health data from far, usually when the patient is at their home. RPM is effective for chronic conditions, such as diabetes, asthma, heart disease, etc. RPM can significantly cut down on the time of patient needs to spend in the hospital and letting them recover at home. With the help of RPM, vital health data can be shared with doctors and other health care professionals remotely. The patients who participate in RPM, have lower chances of experiencing hospital stay, fewer visits to emergency care, improved physical stamina, improved symptom management and, greater emotional well-being and overall satisfaction.

* "Store-and-Forward Practices or Asynchronous Video: Helps patient records and medical data more accessible across long distances. The biggest advantage of this is that it doesn't require the simultaneous attention of the delivering and receiving parties. All sorts of medical imaging, test results, bio-signals can be acquired and transmitted across vast distances. This platform is used by many patient-focused telemedicine platforms to solve minor medical issues.

\section{TELEMEDICINE BENEFITS}

Telemedicine is an alternative benefit for patients and providers alike. It is most successful when a person seeks care from a qualified physician and provides clear details about the symptoms.

- Improves the quality-of-care delivery

- Reduced exposure to pathogens

- Easy access to specialist

- Lower costs

- Medical access to people in rural areas and underserved urban areas

- Reduced medical overhead cost

- On-demand options

- No transportation time or cost

- Less waiting time

- Support for people with chronic disease 
- Disease surveillance and program tracking

- Provides the opportunity for standardization and equity in the provision of healthcare, both within individual countries and across regions and continents.

\section{CHALLENGES}

A survey of health organization was conducted by American Telemedicine Association (ATA) in 2017, identified the following challenges expected in the next three years:

$\star \quad$ Inadequate coverage and payments

$\star \quad$ Licensure and privileges

$\star$ Resistance to change

$\star$ Lack of ROI and quality evidence

$\star$ Tele health provider recruitment

$\star$ Legal Liability

$\star$ Bandwidth limitations

$\star$ Privacy/Cyber security

\section{POTENTIAL DEMAND}

The Global Telemedicine market size was USD 41.63 billion in 2019. The global impact of Covid-19 has been unparalleled and astonishing, with telemedicine witnessing a positive demand shock all over the world. Based on the analysis, the global market will exhibit a stellar growth of $91.7 \%$ in 2020 . The Global Telemedicine market is expected to grow at an annual growth rate of $22.4 \%$ from 2021 to 2028 to reach USD 298.9 billion by 2028. Any disruptive healthcare innovation, takes time, validation and, the right catalyst before it comes fully embraced across the medical community. But with the coronavirus pandemic, the one innovation at the forefront in the transformation of the healthcare landscape is telemedicine. The following illustrates the impact of Telemedicine in the future.

$>$ Telemedicine will become a standard service offered across all care settings: Patients are becoming accustomed to the level of access telemedicine provides. "The Box Is Open". The continued growth of telehealth will be sustained for the years to come. The common thread will be easier access to healthcare, which will generate confidence and drive growth.

$>$ Patients will choose providers, health systems, and hospitals based on telemedicine access: "Telemedicine will redefine patient expectations in all facets of quality healthcare". When patients feel the care of telemedicine better than the routine visit in person, all from the comfort of home, patients will no longer endure long wait times when it is avoidable. As the patients are accustomed to virtual care, there will be a decreased volume of patients for providers that lack telemedicine access.

> Medical facilities that embrace telemedicine will see business and revenue growth: Now and in the future. Telemedicine is a revenue source that can safeguard against future troughs in healthcare. Patients are requesting telemedicine. The volumes and business increase significantly because of the pandemic.

$>$ Telemedicine will become an efficient option for preventative care: According to the Centers for Disease Control and Prevention, chronic diseases that are avoidable through preventive care services account for $75 \%$ of the nation's healthcare spending. By offering Telemedicine services for follow-up care, specialists for faster diagnosis and treatment, hospitals will demonstrate fewer readmissions, complications, inpatient stays and reduce higher cost treatments and services. The goal of future care is preventive.

$>$ Access to specialists will become the norm, which will benefit hospital wait times: As technology innovations evolve healthcare and patient expectations, preparations become more critical. With immediate access to a wide range of specialists, patients will get focused care which will improve the overall patient's experience and reduce the cost of hiring full-time staff on-site.

$>$ Increasing Acceptance of Telehealth as a Trend in Standard of Care: Medical imaging was the first field to accept telemedicine trends. Medical professionals relied on the long-distance transmission of imaging. This practice is common today and it's become a de facto standard in almost all providers.

$>$ Reduced Reliance on Reimbursement Models: In the past reimbursement was the standard way to pay for telemedicine, but it's likely to change moving forward. Healthcare is no longer provided just by independent physicians and institutions. Modern professionals are typically governed by accountable care organizations, managed care groups that account for more than 70 million patients in the United States.

$>$ International Collaboration: The use of telemedicine across national borders was relatively rare. But now the worldwide data transmission networks are much sturdier and medical regulations have 
grown familiar with telemedicine, and the infrastructure is in place to deliver much better medical care to foreign countries.

> Peer Sharing Instead of Hub Sharing: In the past, all the networks utilized a centralized "hub" model in which information was collected from a wide area and sent to major hospitals. In the future, more and more communication will occur directly between individual health care facilities. These peer-topeer networks are popping up in which equal partners join together to share their remote medical resources.

$>$ Mobile Health: This is one of the most exciting new trends in telemedicine. It is also known as mHealth. The possibility of widely distributing medical information to practitioners and patients using mobile technology is extremely promising. The continuing growth of wireless communication technology will bring mHealth initiatives to maturity in the future.

$>$ Virtual Medical Centers: There are several exciting pilot programs in place to distribute the value of centralized specialists across a wider geographic area.

$>$ New Remote Clinical Services: There are already several vendors providing remote care in the fields of radiology, dermatology, mental health, and stroke care. Hospitals are getting into it leading to a volatile and competitive market.

Some of the major findings of the future of Telemedicine include:

$>$ The growing importance of social distancing and self-quarantine will lead to an increase in the use of telemedicine

$>$ Increasing cases of Covid-19 pandemic across the globe and the growing influence of social distancing will drive the market

$>$ The neurology segment will witness significant growth across the forecast time frame

$>$ Telemonitoring services will be increasingly adopted to lower the burden on the medical fraternity

$>$ Minimizing the spread of Covid-19 infection among the healthcare staff will lead to growth in the telemedicine growth

$>$ Increasing need to lower the overall healthcare cost and technological advancements will be conducive to the market growth

$>$ Competitors will start focusing on expanding the capability of managing the high flux of calls, mergers, CONCLUSION and collaborations in a bid to capture market share and strengthen revenue generation.

The pandemic quickly proved that telemedicine could be a lifeline for practices. Telemedicine is breaking down barriers to receiving care - time, distance, and availability are becoming smaller obstacles. Telehealth connects healthcare professionals and patients promptly, conveniently, and efficiently. As the healthcare leaders work with the lawmakers to remove the red tape and to broaden the use of telehealth, healthcare professionals will be preparing for and adapting to the positive changes to come. The future of telemedicine, in which people spend less time traveling to and from healthcare facilities while still receiving high-quality care virtually from their homes and other remote locations.

"Telemedicine improves the quality-of-care delivery". 


\section{ARTIC LES}

\section{REFERENCE}

1. https://yourstory.com/2020/12/india-recorded-3x-increase-online-consultations-practo-report

2. https://yourstory.com/2021/01/telemedicine-growth-india-healthcare-future-ready

3. https://chironhealth.com/definitive-guide-to-telemedicine/telemedicine-info-patients/advantagestelemedicine-patients/

4. https://www.healthline.com/health/healthcare-provider/telemedicine-benefits

5. https://health.economictimes.indiatimes.com/news/health-it/outlook-2019-the-future-oftelemedicine-in-india/67366188

6. https://www.beckershospitalreview.com/how-video-is-shaping-the-future-of-telemedicine.html

7. https://www.managedhealthcareexecutive.com/view/how-much-will-telehealth-go-back-to-thefuture-following-the-pandemic-

8. https://online.maryville.edu/blog/the-future-of-telehealth/

9. https://www.modernhealthcare.com/technology/predicting-future-role-telemedicine

10. https://evisit.com/resources/future-of-telemedicine-7-trends-shaping-the-future-of-telehealth/

11. https://consultqd.clevelandclinic.org/the-future-of-telemedicine-and-whats-in-the-way/

12. https://online.maryville.edu/blog/the-future-of-telehealth/

13. https://www.medicaleconomics.com/view/digital-doctors-what-role-will-telehealth-play-after-covid19-

14. https://www.managedhealthcareexecutive.com/view/how-much-will-telehealth-go-back-to-thefuture-following-the-pandemic-

15. https://www.hindawi.com/journals/ddns/2019/7642176/?utm_source=google\&utm_medium=cpc\&u tm_campaign=HDW_MRKT_GBL_SUB_ADWO_PAI_DYNA_JOUR_X_PCUPS\&gclid=EAlalQobChMI2qnR wOWd8AIVCtTtCh0Q-AVFEAMYAiAAEgLPHvD_BwE

16. https://www.fortunebusinessinsights.com/industry-reports/telemedicine-market-101067

17. https://content.iospress.com/articles/journal-of-parkinsons-disease/jpd202109 